\title{
Assessment of rheumatic mitral valve disease Value of echocardiography in patients clinically suspected of predominant stenosis
}

\author{
H EGEBLAD, J BERNING, K SAUNAMÄKI, J R JACOBSEN, A WENNEVOLD \\ From Cardiovascular Laboratory of Medical Department B, Rigshospitalet, Copenhagen, Denmark
}

SUMMARY The value of echocardiography as compared with cardiac catheterisation was evaluated prospectively in 33 consecutive patients clinically suspected of predominant mitral stenosis. Patients with clinical signs of accompanying mitral regurgitation, no matter how severe, and patients with clinical findings indicating insignificant aortic valve disease were included. Critical mitral stenosis was defined by a valve area of $\leqslant 1 \mathrm{~cm}^{2}$. Severe mitral regurgitation was diagnosed by echocardiography on the basis of left ventricular dilatation (more than $3.2 \mathrm{~cm} / \mathrm{m}^{2}$ at end-diastole) if not explained otherwise. Significant aortic valve disease was suspected in cases with aortic valve deformity and left ventricular dilatation or hypertrophy as defined by echocardiography.

Mitral valve area by echocardiography correlated well with mitral valve area calculated from catheterisation data and a good interobserver correlation was found for echocardiographic measurement. Mitral stenosis, critical or non-critical, may mask significant coexistent valve lesions; echocardiography failed to discover severe mitral regurgitation requiring valve replacement in two patients with non-critical stenosis, and significant aortic regurgitation needing valve replacement was underestimated in one patient with critical mitral stenosis. A correct echocardiographic classification with respect to surgery, however, was obtained in: (1) all patients with clinically pure mitral stenosis (nine patients), and (2) all patients with combined mitral stenosis and regurgitation when either critical stenosis or severe regurgitation was found at echocardiography (12 patients).

It thus appears that two out of three patients with mitral valve disease in whom the clinical findings indicate predominant stenosis can be correctly evaluated with the echocardiogram.

The value of $M$-mode echocardiography in the diagnosis of rheumatic mitral valve disease has been recognised for a number of years. ${ }^{2}$ Indirect estimation of the mitral valve area has been attempted by measurement of leaflet motion, by analysis of the emptying pattern of the left atrium, and by determination of the left ventricular filling rate. ${ }^{3-5}$ Two dimensional echocardiography has considerably refined the assessment of rheumatic mitral valve disease by offering a tomographic visualisation of the entire mitral apparatus with delineation of the mitral orifice proper. Recent reports have documented excellent correlations between mitral valve area as determined by two dimensional echocardiography and the orifice size measured during operation ${ }^{6}$ or calculated on the basis of catheterisation data. $^{7-9}$ Accordingly, there is a growing feeling that echocardiography might replace cardiac catheterisation in the evaluation of patients with mitral

Accepted for publication 17 August 1982 stenosis. ${ }^{10}{ }^{11}$ In patients with mitral stenosis, however, the situation is often influenced by associated lesions, for example mitral regurgitation, aortic valve disease, and myocardial dysfunction and these lesions may in themselves necessitate cardiac surgery.

In the present study, the value of echocardiography in the determination of mitral valve area and in the evaluation of coexistent lesions was analysed in a group of consecutive patients clinically suspected of isolated or predominant mitral stenosis.

\section{Patients and methods}

During a one year period, consecutive patients suspected on the basis of clinical findings, electrocardiogram, and chest $x$-ray examination of severe and predominant rheumatic mitral valve stenosis were included in the study. Patients with clinical findings indicating a coexistent severe aortic valve lesion or 
ischaemic heart disease were excluded, whereas patients with clinical signs of concomitant mild aortic valve disease thought unlikely to require valve replacement and all patients with murmurs indicating combined mitral stenosis and regurgitation remained in the study. The criteria used were: (1) incapacitating exertional dyspnoea affecting the social situation of the patient; (2) the presence of a typical low frequency diastolic rumbling murmur at the apex with or without an accompanying pansystolic blowing murmur with radiation to the left axilla; (3) no diastolic high frequency early diastolic decrescendo murmur at the left sternal border louder than grade 1 ; (4) no ejection murmur or only a grade 1 to 2 short systolic ejection murmur at the base of the heart with a distinct aortic second heart sound; (5) a normal carotid pulse, normal blood pressure amplitude, and no electrocardiographic signs of left ventricular hypertrophy or strain in the presence of an aortic valve murmur; (6) no history or electrocardiographic findings indicative of ischaemic heart disease; (7) a cardiac silhouette indicating mitral valve disease but without dilatation of the ascending aorta on chest $x$-ray.

Selection of patients for the study according to these criteria was carried out by a single experienced cardiologist. The study population consisted of 33 patients ( 29 women and 4 men) with a mean age of 51 years (range 21 to 69 years).

Echocardiography was performed with an Aloka $^{\mathrm{R}}$ SSD $110 \mathrm{~S}-\mathrm{E}$ equipment the day before heart catheterisation. M-mode echocardiograms $(2.25 \mathrm{MHz}$ transducer) were obtained with the use of a fibreoptic recorder. Two dimensional echocardiography was carried out with the mechanical sector scanner of the Aloka ${ }^{\mathrm{R}}$ ultrasonic apparatus using either a $2 \cdot 25$ or a 3 $\mathrm{MHz}$ transducer. Records were stored on video tape employing a recorder with options for replay in slow motion and for stop frames. During replay the video recorder was connected to a Cardio 80 (Kontron ${ }^{\mathrm{R}}$ Inc.) graph pen computer system.

Left ventricular end-diastolic and end-systolic diameters were measured as averages of the measurements of three to four consecutive cardiac cycles recorded by $\mathrm{M}$-mode echocardiography according to conventional methods. ${ }^{12}$ Delineation of septal and posterior wall endocardium at end-diastole and endsystole but not necessarily throughout the cardiac cycle was required for measurement.

The orientation of the mitral valve orifice was established by two dimensional echocardiography using multiple parasternal sections in the long axis view. This determination ensured optimal position of the transducer for subsequent scanning of the mitral valve orifice in the short axis view. ${ }^{9}$ Care was taken to avoid inappropriately high or low gain settings according to Martin et al. ${ }^{9}$ Using the electronic pen of the computer system, the black and white interface of the mitral orifice was outlined according to Martin et al. ${ }^{9}$ (Fig. 1). The procedure was repeated five times for each of two randomly selected heart cycles and the mean value was computed. Complete mitral valve area measurement was carried out by two independent observers in each patient in order to evaluate the interobserver variability.

Severe mitral regurgitation was defined by: (1) a murmur indicating mitral regurgitation; (2) volume load of the left ventricle, that is dilatation of the enddiastolic diameter to more than $3.2 \mathrm{~cm} / \mathrm{m}^{2}$ body surface area $^{12}$ in the face of increased or normal fractional shortening of the left ventricle ( 25 to $40 \%$ ); and (3) no other clinical or echocardiographic finding that might account for left ventricular dilatation.

In patients with clinical findings indicating mild aortic valve disease, a significant aortic valve lesion necessitating valve replacement was designated as suspected if the echocardiogram showed aortic valve abnormalities in combination with left ventricular

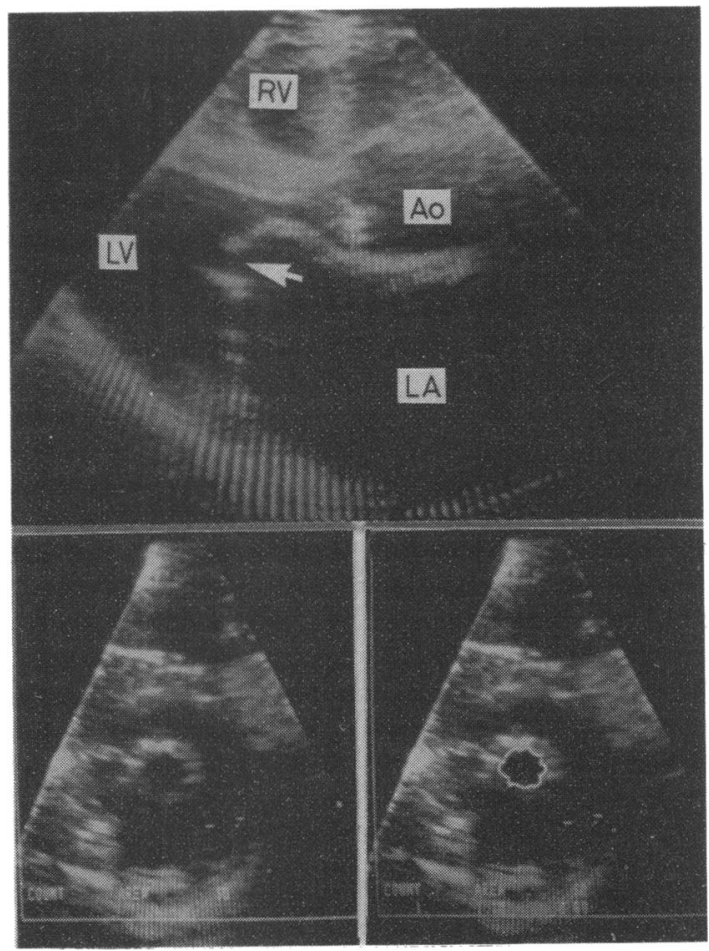

Fig. 1 Upper panel: parasternal long axis view showing mitral orifice in early diastole (arrow). Lower panels: parasternal short axis view of the mitral orifice (early diastole); calibration grid is faintly seen. In the lower right panel the valve area has been outlined by use of the graph pen and the numerical value of the mitral valve area is indicated at the bottom $\left(1 \cdot 15 \mathrm{~cm}^{2}\right)$. Ao, aortic root; $L A$, left atrium; $L V$, left ventricle; $R V$, right ventricle. 
dilatation or hypertrophy (end-diastolic septal and posterior wall thickness of more than $1 \cdot 1 \mathrm{~cm}^{12}$ ).

Cardiac catheterisation was performed by the percutaneous Seldinger technique from the groin. The diastolic mean gradient across the mitral valve was obtained by planimetry of the area between calibrated pressure curves from a pulmonary wedge position and left ventricle, respectively. In patients with combined mitral stenosis and regurgitation, mitral flow was determined by the Fick method with correction for the regurgitant fraction (see below). Mitral valve area was calculated with the use of the Gorlin formula. ${ }^{1314} \mathrm{~A}$ mitral valve area of $1 \mathrm{~cm}^{2}$ or less was considered a critical stenosis necessitating surgical treatment.

Right anterior oblique cineventriculography was done in all patients except case 5. Left anterior oblique cineaortography was performed in patients with a murmur indicating aortic regurgitation and occasionally in patients without definite clinical signs of aortic valve disease. Both mitral and aortic valve regurgitation were graded on a scale from I to IV according to conventional criteria. ${ }^{15} 16$ The regurgitant fraction was calculated as: Stroke volume Angio $_{\text {- }}$ stroke volume $_{\text {Fick }} /$ stroke volume $_{\text {Angio }} \times 100 \%$. A regurgitant fraction of $40 \%$ or more was considered haemodynamically important and indicating severe mitral or aortic valve regurgitation. ${ }^{17}$

In cases with combined mitral and aortic valve regurgitation each of grade II or more, neither the stroke volume by the Fick method nor the stroke volume corrected by quantitative angiography were considered valid for use in the Gorlin formula. Regurgitation of grade 1 of either valve was disregarded as negligible for the calculations in combined mitral and aortic regurgitation.

Coronary arteriography was not performed in any of the patients. Echocardiography and cardiac catheterisation were carried out and analysed by independent examiners who were unaware of each other's results.

Echocardiographic results were not used in making decisions about surgery.

\section{Results}

Clinical and echocardiographic data, and results from cardiac catheterisation and angiography are presented in Fig. 2 and Table 1. For the total series (apart from cases 14,31 , and 33 , in whom only incomplete catheterisation data for reference purposes were available because of combined grade II mitral and aortic regurgitation) a linear correlation between mitral valve area as determined by echocardiography and by cardiac catheterisation was demonstrated $(r=0.91,0.84-0.95$, with $95 \%$ confidence limits $(p<0.01)$; standard error of the estimate $(\mathrm{SEE})=0.28 \mathrm{~cm}^{2}$ ). Comparison of the echocardiographic measurement of mitral valve area by two independent observers gave a linear correlation:

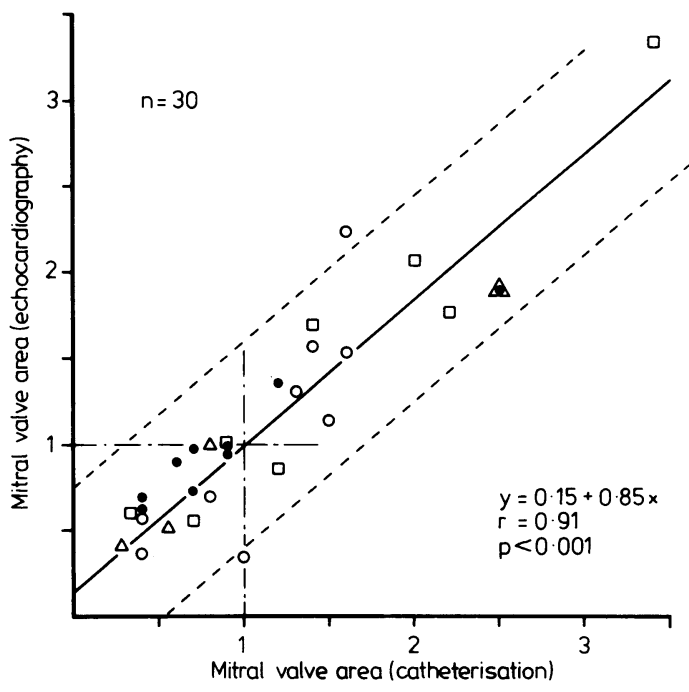

Fig. 2 Mitral valve area as determined by echocardiography $\left(\mathrm{cm}^{2}\right)$ (observer no I) depicted versus valve area as calculated from catheterisation data $\left(\mathrm{cm}^{2}\right)$ in 30 consecutive patients suspected of severe mitral stenosis. Closed circles, clinical pure mitral stenosis. Open circles, mitral stenosis with coexistent lesions suspected on the basis of echocardiography to be negligible. Squares, mitral stenosis combined with mitral regurgitation suspected on the basis of echocardiography to be severe. Triangles, mitral stenosis with coexistent severe aortic valve lesions on the basis of echocardiography. Regression line with $\pm 2 S E E$ is indicated. In addition, cut-off level of critical mitral stenosis is marked.

Area $_{\mathrm{I}}=0.23+0.73 \times$ Area $_{\mathrm{II}} ; \quad \mathrm{r}=0.89 \quad(0.79-0.94)$, $\mathrm{p}<0.01 ; \mathrm{SEE}=0.19 \mathrm{~cm}^{2}$.

On the basis of the clinical and echocardiographic findings the patients were classified into four groups (Table 1).

\section{GROUP 1}

Nine patients (cases 1 to 9) had murmurs and echocardiograms indicating pure mitral stenosis. No sign of coexistent lesions was found by subsequent invasive study in these patients. Angiography was omitted in case 5 because of a recent episode of spontaneous ventricular fibrillation. Cases 1 to 7 were referred for valve replacement because of critical mitral stenosis.

\section{GROUP 2}

Ten patients (cases 10 to 19) had murmurs indicating mitral stenosis combined with mitral regurgitation and/or mild aortic valve disease. As there was no echocardiographic left ventricular dilatation, left ventricular hypertrophy, or aortic valve abnormality apart from a mild increase of valvular echo density, these patients were considered to have mitral valve stenosis with minor coexistent lesions. At catheterisation, however, this conclusion appeared incorrect in 
Table 1 Clinical findings, and echocardiographic and heart catheterisation data in 33 consecutive patients with suspected critical mitral stenosis

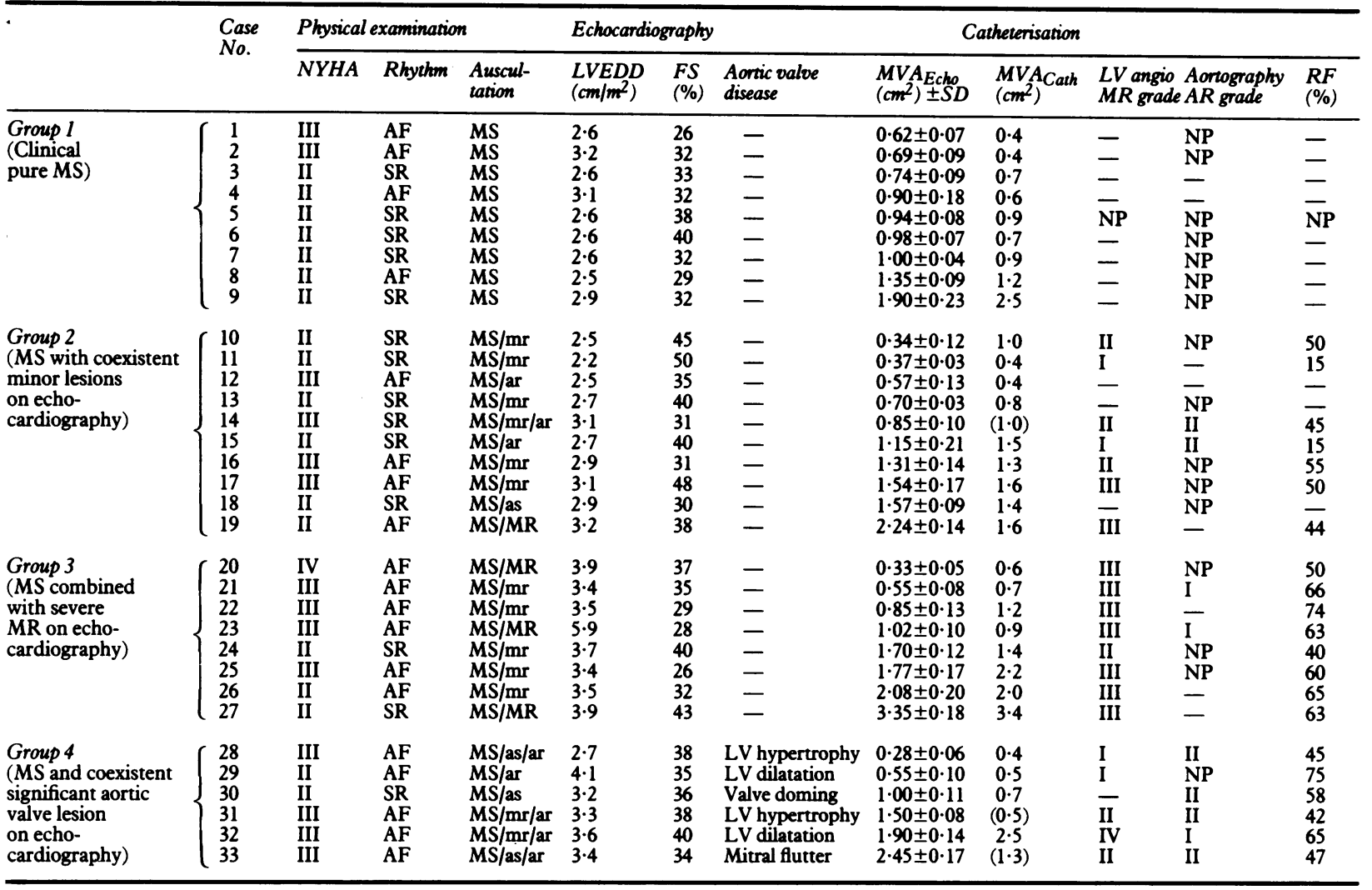

Patients were classified into groups 1 to 4 based on clinical findings and echocardiography. AF, atrial fibrillation; Aortic valve disease, echocardiographic signs of significant aortic valve lesion apart from cusp thickening; Aortography AR grade, grade I-IV of aortic valve regurgitation as estimated from cineaortography; ar, mild aortic regurgitation; as, mild aortic stenosis; FS, fractional shortening of the left ventricle; LV angio MR grade, grade I-IV of mitral regurgitation as evaluated from left ventricular angiography; LVEDD, left ventricular end-diastolic diameter; $\mathrm{mr}$, mild mitral regurgitation; $M R$, severe mitral regurgitation; $M$ S, mitral stenosis; NP, not performed; NYHA, functional class I-IV according to the criteria of the New York Heart Association; RF, regurgitant fraction; SR, sinus rhythm; -, absent. Mitral valve area (MVA) in values in brackets were obtained by the Fick method in patients with combined moderate mitral and aortic regurgitation; true valve area could not be calculated from catheterisation data in these patients.

five patients. Case 10 , with critical mitral stenosis, had severe concomitant mitral regurgitation on angiography. Case 14 with critical mitral stenosis as determined by echocardiography also had grade II mitral regurgitation and grade II aortic regurgitation. From the catheterisation data and operative findings, this patient was found to need both mitral and aortic valve replacement. Cases 16 and 17 with non-critical mitral stenosis had coexistent mitral regurgitation of grade II and III with regurgitant fractions of $55 \%$ and $50 \%$, respectively, requiring mitral valve replacement in both cases. Case 19 also had severe mitral regurgitation as evaluated by angiography, but operation was indefinitely postponed because of limited symptoms and because of the patient's resistance to the proposal. In the remaining five patients the echocardiographic findings were confirmed by catheterisation.
In group 2, all patients except for cases 15,18 , and 19 were referred for mitral valve replacement; case 14 had combined mitral and aortic valve replacement.

GROUP 3

Echocardiograms of eight patients (cases 20 to 27) with murmurs indicative of combined mitral stenosis and regurgitation all had echocardiographic volume overload of the left ventricle, indicating severe mitral regurgitation according to our criteria. Angiography confirmed the presence of severe regurgitation in every patient, each of whom underwent subsequent mitral valve replacement.

\section{GROUP 4}

Six patients (cases 28 to 33 ) had murmurs of combined mitral and aortic valve disease with echocardiographic 
findings indicating significant aortic valve involvement. Cases 28 and 31 both had diffuse left ventricular hypertrophy.

Case 29 had left ventricular volume overload which in the light of the auscultatory findings was considered to indicate significant aortic regurgitation. Left ventricular angiography showed only grade I mitral regurgitation which appeared to be incompatible with the regurgitant fraction of $75 \%$ shown in this patient. Furthermore, left ventricular angiography suggested aortic regurgitation because of the impression of the flow of contrast medium to and fro between the left ventricle and the aortic root. Unfortunately, aortography had to be omitted because of severe discomfort after left ventricular angiogram. Later, during open heart surgery, indirect signs of aortic regurgitation were shown, but the aortic valve was not explored. This patient was excluded from the subsequent analysis because of inadequate assessment of the severity of the aortic regurgitation at catheterisation and operation. From the data available, however, it seems that the echocardiographic suggestion of significant aortic regurgitation may well have been correct.

Case 30 had borderline left ventricular dilatation and hypertrophy but definite doming of the aortic valve cusps. Case 32 had murmurs compatible with both mitral and aortic regurgitation, and echocardiography showed volume overload of the left ventricle. Accordingly, the patient was classified as having possibly significant aortic regurgitation since echocardiography could not differentiate between the relative significance of the mitral and aortic regurgitation; on angiography mitral regurgitation was shown to be the dominant of the two lesions.

The diastolic murmur at the apex in case 33 may have been an Austin-Flint murmur since only mild mitral valve obstruction was found; echocardiography showed left ventricular dilatation and mitral valve high frequency flutter indicating aortic valve regurgitation.

Thus, excluding case 29, four of the five patients (cases 28, 30, 31, and 33) with echocardiographic findings of mitral stenosis and significant coexistent aortic valve disease had such lesions documented by catheterisation, and all four patients underwent subsequent combined mitral and aortic valve replacement. Case 32 underwent mitral valve replacement alone. At catheterisation none of the patients in the series had systolic peak gradients across the aortic valve exceeding $10 \mathrm{mmHg}$. Open heart surgery, however, indicated significant aortic stenosis in case 30 in accordance with the echocardiographic findings.

\section{Discussion}

The tomographic display of the mitral orifice by two dimensional echocardiography offers an attractive means of measuring mitral valve area as opposed to conventional indirect calculation of the valve area based on catheterisation data. The accuracy of the two dimensional echocardiographic measure of mitral valve area depends primarily on correct transducer angulation and on the gain setting of the ultrasonic equipment, factors difficult to standardise. ${ }^{9}{ }^{18}$ Accordingly, experience with the echocardiographic technique improves the results. ${ }^{19}$ Computation of valve area from the Gorlin formula is based on various measurements, each associated with some inaccuracy. Taking these imperfections of both methods into consideration, recent studies have shown that mitral valve area can be measured reliably by two dimensional echocardiography in patients with pure mitral stenosis and with combined mitral stenosis and regurgitation. 6-8 $^{-8}$ Knowledge of the size of mitral valve area does not always sufficiently delineate the condition, but, in the determination of mitral stenosis, echocardiography has further potential: the degree of mitral regurgitation may be reflected by the left ventricular volume load; aortic valve morphology and mobility are visualised; indirect information of the degree of aortic valve disease is provided by evaluation of the left ventricular cavity size and wall thickness; and complicating localised or global myocardial dysfunction may be detected.

In our series, all patients had echocardiograms technically adequate for analysis of the mitral orifice, the aortic valve, and the left ventricle. A lower rate of success with respect to mitral valve visualisation had been found in previous studies. ${ }^{78}$ Admittedly, technical difficulties may occur in the occasional patient because of obesity or emphysema, but such limitations are not usually of major importance, and the problems of cardiac catheterisation must be kept in mind. Two of our patients had incomplete catheterisation caused either by anticipated or experienced adverse reactions during the injection of contrast medium.

The interobserver correlation in our series and in a previous study indicates high reproducibility of the echocardiographic measurement of mitral valve area. ${ }^{9}$ The correlation between the valve area as determined by catheterisation data and by echocardiography found in the present and in previous studies indicates that mitral valve area can be measured with acceptable accuracy by echocardiography in the individual patient within the spectrum of mitral valve disease. Worthy of attention are the patients suspected by clinical and echocardiographic examination of having mitral stenosis and additional minor valve lesions (patients in group 2, Table 1). In three of these patients with non-critical stenosis echocardiography did not detect severe mitral regurgitation, and in one case severe coexistent aortic regurgitation was underestimated. Echocardiographic findings indicating negligible mitral regurgitation in patients with non-critical 
stenosis and mild aortic valve disease in patients with mitral stenosis should, therefore, be treated with caution. Not unexpectedly, mitral stenosis in some patients may mask significant valve regurgitation, probably by protecting the left ventricle from early diastolic volume overload. This concept is in accordance with the observation that the left ventricular enddiastolic cavity diameter is often small in patients with pure mitral stenosis; it is also consistent with the finding in the present study that even minor left ventricular volume overload on echocardiography reflected severe mitral regurgitation in the absence of other lesions causion left ventricular dilatation.

No patient in our series had left ventricular dilatation combined with myocardial hypokinesia. Such a finding might reflect secondary myocardial injury caused by long standing severe mitral regurgitation, but should arouse suspicion of ischaemic or primary myocardial disease and lead to further investigation.

Ten patients in the present series had clinical findings indicating mitral stenosis with or without regurgitation in combination with mild aortic valve disease. Most of these patients were correctly classified by echocardiography as having either insignificant or severe coexistent aortic valve lesions. One patient, however, had echocardiographic signs of mild aortic regurgitation, but was later found to need both mitral and aortic valve replacement. Two patients with murmurs indicating combined aortic and mitral valve regurgitation had echocardiograms demonstrating left ventricular dilatation; our criteria did not allow a distinction between the relative significance of the incompetence of the valves involved. Using these criteria, therefore, it seems advisable to perform both echocardiography and cardiac catheterisation in patients with signs of combined mitral and aortic valve disease. Angiography appears adequate for defining the relative degree of the valve incompetence, whereas echocardiography provides the mitral valve area which cannot reliably be determined by invasive examination in patients with moderate or severe combined mitral and aortic regurgitation.

Thus, it appears that mitral valve area can be adequately measured by two dimensional echocardiography in the kind of patients examined. A correct classification with respect to the need for surgical correction seems feasible (1) in patients with clinical findings indicating pure mitral stenosis, and (2) in patients with clinical findings indicating combined mitral stenosis and regurgitation and with echocardiographic findings of either critical stenosis or severe regurgitation. In the present series, such patients constituted 21 out of the 33 patients with mitral stenosis. Therefore, $21 / 33$ (64\%) (45-80\% with $95 \%$ confidence limits) of patients with clinically predominant mitral stenosis can be adequately evaluated by combined clinical and echocardiographic examination. The likelihood of obtaining identical therapeutic conclusions by echocardiography and cardiac catheterisation in the individual categories of patients is presented in Table 2 .

Measurement of pulmonary pressure during rest and exercise may be of importance in patients who display significant disparity between symptoms and echocardiographic findings. Such data as well as information concerning the coronary arteries cannot be

Table 2 Echocardiography and heart catheterisation: comparison of therapeutical conclusions based on information on severity of mitral stenosis and associated lesions

\begin{tabular}{|c|c|c|c|}
\hline Patients & $\begin{array}{l}\text { Significant under- } \\
\text { estimation by echo } \\
\text { Case No. and lesion }\end{array}$ & $\begin{array}{l}\text { Identical consequence } \\
\text { echo/catheterisation } \\
\text { No. } \%(95 \% \text { confidence limits) }\end{array}$ & $\begin{array}{l}\text { Significant over- } \\
\text { estimation by echo } \\
\text { Case No. and lesion }\end{array}$ \\
\hline $\begin{array}{l}\text { Group I } \\
\text { (Clinical pure MS) } \\
(n=9)\end{array}$ & - & $9100(66-100)$ & - \\
\hline $\begin{array}{l}\text { Group } 2 \\
\text { (MS with coexistent } \\
\text { minor lesions on } \\
\text { echocardiography) } \\
\quad(n=10)\end{array}$ & $\begin{array}{l}\text { Case } 14, \text { AR } \\
\text { Cases } 16 \text { and } 17, \text { MR } \\
\text { (Case } 19, M R \text { on angio, but no } \\
\text { valve replacement performed) }\end{array}$ & $7 \quad 70(35-93)$ & - \\
\hline $\begin{array}{l}\text { Group } 3 \\
\text { (MS combined with severe } \\
\text { MR on echocardiography) } \\
\quad(n=8)\end{array}$ & - & $8 \quad 100(63-100)$ & - \\
\hline $\begin{array}{l}\text { Group } 4 \\
\text { (MS and coexistent } \\
\text { significant aortic valve } \\
\text { lesion on echocardiography) } \\
\quad(n=6)\end{array}$ & - & $4 \quad 80(28-99)$ & $\begin{array}{l}\text { Case } 29 \text { excluded } \\
\text { (see results) } \\
\text { Case 32, possible AR }\end{array}$ \\
\hline
\end{tabular}

Patients are classified as in Table 1. In the middle column are listed the number and percentage of patients in whom identical conclusions were drawn with respect to the need for surgical correction. AR, severe aortic regurgitation; MR, severe mitral regurgitation; MS, mitral stenosis. 
obtained by echocardiography. Therefore echocardiography should be regarded as a substitute for catheterisation only in typical cases. Otherwise, the two techniques should be considered complementary at present.

\section{References}

1 Edler I. Ultrasoundcardiography in mitral valve stenosis. Am f Cardiol 1967; 19: 18-31.

2 Cope GD, Kisslo JA, Johnson ML, Behar VS. A reassessment of the echocardiogram in mitral stenosis. Circulation 1975; 52: 664-70.

3 Shiu MF. Mitral valve closure index. Echocardiographic index of severity of mitral stenosis. Br Heart $\mathcal{F} 1979$; 39: 839-43.

4 Naccarelli GV, Nomeir AM, Watts LE, Zelis R. Echocardiographic assessment of mitral stenosis by the left atrial emptying index. Chest 1979; 76: 668-71.

5 Wise JR Jr. Echocardiographic evaluation of mitral stenosis using diastolic posterior left ventricular wall motion. Circulation 1980; 61: 1037-42.

6 Henry WL, Griffith JM, Michaelis LL, McIntosh CL, Morrow AG, Epstein SE. Measurement of mitral orifice area in patients with mitral valve disease by real-time two-dimensional echocardiography. Circulation 1975; 51: 827-31.

7 Nichol PM, Gilbert BW, Kisslo JA. Two-dimensional echocardiographic assessment of mitral stenosis. Circulation 1977; 55: 120-8.

8 Wann LS, Weyman AE, Feigenbaum H, Dillon JC, Johnston KW, Eggleton RC. Determination of mitral valve area by cross-sectional echocardiography. Ann Intern Med 1978; 88: 337-41.

9 Martin RP, Rakowski H, Kleiman JH, Beaver W, London E, Popp RL. Reliability and reproducibility of two-dimensional echocardiographic measurement of the stenotic mitral valve orifice area. Am $\mathcal{F}$ Cardiol 1979; 43: 560-8.

10 Motro M, Neufeld HN. Should patients with pure mitral stenosis undergo cardiac catheterization? Am $\mathcal{f}$ Cardiol 1980; 46: 515-6.

11 St John Sutton MG, St John Sutton M, Oldershaw P, et al. Valve replacement without preoperative cardiac catheterisation. N Engl f Med 1981; 305: 1233-8.

12 Feigenbaum H. Echocardiography. 3rd ed. Philadelphia: Lea \& Febiger, 1981: 550

13 Gorlin R, Gorlin SG. Hydraulic formula for calculation of the area of the stenotic mitral valve, other cardiac valves and central circulating shunts. 1. Am Heart f 1951; 41: 1-29.

14 Cohen MV, Gorlin R. Modified orifice equation for the calculation of mitral valve area. Am Heart $\mathcal{F} 1972 ; 84$ : 839-40.

15 Sellers RD, Levy MJ, Amplatz K, Lillehei W. Left retrograde cardioangiography in acquired cardiac disease. $\mathrm{Am}$ f Cardiol 1964; 14: 437-47.

16 Hurst JW, Logue RB, eds. The heart. 3rd ed. New York: McGraw-Hill, 1974: 872.

17 Braunwald E. Heart disease: a textbook of cardiovascular medicine. Philadelphia: Saunders, 1980: 294 \& 1116.

18 Naito M, Morganroth J, Mardelli TJ, Chen CC, Dreifus LS. Rheumatic mitral stenosis: cross-sectional echocardiographic analysis. Am Heart f 1980; 100: 34-40.

19 Jain SK, Pechacek LW, DeCastro CM, Garcia E, Hall RJ. Non-invasive assessment of the stenotic mitral orifice by two-dimensional echocardiography. Cardiovasc Dis Bull Texas Heart Inst 1981; 8: 29-38.

Requests for reprints to Dr Henrik Egeblad, Medical Department B, Rigshospitalet, Blegdamsvej 9, 2100DK Copenhagen $\emptyset$, Denmark. 